\title{
On selective sequential separability of function spaces with the compact-open topology
}

\author{
Alexander V. Osipov (iD \\ Krasovskii Institute of Mathematics and Mechanics, Ural Federal University, Ural State University of \\ Economics, 620219, Ekaterinburg, Russia
}

\begin{abstract}
For a Tychonoff space $X$, we denote by $C_{k}(X)$ the space of all real-valued continuous functions on $X$ with the compact-open topology. A subset $A \subset X$ is said to be sequentially dense in $X$ if every point of $X$ is the limit of a convergent sequence in $A$. A space $C_{k}(X)$ is selectively sequentially separable (in Scheepers' terminology: $C_{k}(X)$ satisfies $S_{f i n}(\mathcal{S}, \mathcal{S})$ ) if whenever $\left(S_{n}: n \in \mathbb{N}\right)$ is a sequence of sequentially dense subsets of $C_{k}(X)$, one can pick finite $F_{n} \subset S_{n}(n \in \mathbb{N})$ such that $\bigcup\left\{F_{n}: n \in \mathbb{N}\right\}$ is sequentially dense in $C_{k}(X)$. In this paper, we give a characterization for $C_{k}(X)$ to satisfy $S_{f i n}(\mathcal{S}, \mathcal{S})$.
\end{abstract}

Mathematics Subject Classification (2010). 54C25, 54C35, 54C40, 54D20

Keywords. compact-open topology, function space, selectively sequentially separable, $S_{1}(\mathcal{S}, \mathcal{S})$, sequentially dense set, property $\alpha_{2}$, property $\alpha_{4}$

\section{Introduction}

If $X$ is a topological space and $A \subseteq X$, then the sequential closure of $A$, denoted by $[A]_{\text {seq }}$, is the set of all limits of sequences from $A$. A set $D \subseteq X$ is said to be sequentially dense if $X=[D]_{\text {seq. }}$. A space $X$ is called sequentially separable if it has a countable sequentially dense set $[26,27]$.

Let $X$ be a topological space, and $x \in X$. Consider the following collections:

- $\Omega_{x}=\{A \subseteq X: x \in \bar{A} \backslash A\}$;

- $\Gamma_{x}=\{A \subseteq X: x=\lim A\}$.

Note that if $A \in \Gamma_{x}$, then there exists $\left\{a_{n}\right\} \subset A$ converging to $x$. So, simply $\Gamma_{x}$ may be the set of non-trivial convergent sequences to $x$.

Many topological properties are defined or characterized in terms of the following classical selection principles. Let $\mathcal{A}$ and $\mathcal{B}$ be sets consisting of families of subsets of an infinite set $X$. Then:

$S_{1}(\mathcal{A}, \mathcal{B})$ is the selection hypothesis: for each sequence $\left(A_{n}: n \in \mathbb{N}\right)$ of elements of $\mathcal{A}$ there is a sequence $\left\{b_{n}\right\}_{n \in \mathbb{N}}$ such that for each $n, b_{n} \in A_{n}$, and $\left\{b_{n}: n \in \mathbb{N}\right\}$ is an element of $\mathcal{B}$.

$S_{\text {fin }}(\mathcal{A}, \mathcal{B})$ is the selection hypothesis: for each sequence $\left(A_{n}: n \in \mathbb{N}\right)$ of elements of $\mathcal{A}$ there is a sequence $\left\{B_{n}\right\}_{n \in \mathbb{N}}$ of finite sets such that for each $n, B_{n} \subseteq A_{n}$, and $\bigcup_{n \in \mathbb{N}} B_{n} \in \mathcal{B}$.

Email address: OAB@list.ru

Received: 13.05.2018; Accepted: 09.07.2018 
In this paper, by a cover we mean a cover $\mathcal{U}$ with $X \notin \mathcal{U}$.

A cover $\mathcal{U}$ of a space $X$ is called:

- a $k$-cover if each compact subset $C$ of $X$ is contained in an element of $\mathcal{U}$;

- a $\gamma_{k}$-cover if $\mathcal{U}$ is infinite and for each compact subset $C$ of $X$ the set $\{U \in \mathcal{U}: C \nsubseteq U\}$ is finite.

Note that a $\gamma_{k}$-cover is a $k$-cover, and a $k$-cover is infinite. A compact space has no $k$-covers.

For a Tychonoff space $X$, we denote by $C_{k}(X)$ the space of all real-valued continuous functions on $X$ with the compact-open topology. Subbase open sets of $C_{k}(X)$ are of the form $[A, U]=\{f \in C(X): f(A) \subset U\}$, where $A$ is a compact subset of $X$ and $U$ is a non-empty open subset of $\mathbb{R}$. Sometimes we will write the basic neighborhood of a point $f \in C_{k}(X)$ as $\langle f, A, \epsilon\rangle$ where $\langle f, A, \epsilon\rangle:=\{g \in C(X):|f(x)-g(x)|<\epsilon \forall x \in A\}, A$ is a compact subset of $X$ and $\epsilon>0$.

For a topological space $X$ we denote:

- $\Gamma_{k}$ - the family of open $\gamma_{k}$-covers of $X$;

- $\mathcal{K}$ - the family of open $k$-covers of $X$;

- $\mathcal{K}_{c z}^{\omega}$ — the family of countable co-zero $k$-covers of $X$;

- $\mathcal{D}$ - the family of dense subsets of $C_{k}(X)$;

- $\mathcal{S}$ - the family of sequentially dense subsets of $C_{k}(X)$

- $\mathbb{K}(X)$ - the family of all non-empty compact subsets of $X$.

A space $X$ is said to be a $\gamma_{k}$-set if each $k$-cover $\mathcal{U}$ of $X$ contains a countable set $\left\{U_{n}: n \in \mathbb{N}\right\}$ which is a $\gamma_{k}$-cover of $X[9]$.

\section{Main definitions and notation}

- A space $X$ is $R$-separable, if $X$ satisfies $S_{1}(\mathcal{D}, \mathcal{D})$ ([2, Definition 47]).

- A space $X$ is selectively separable ( $M$-separable), if $X$ satisfies $S_{\text {fin }}(\mathcal{D}, \mathcal{D})$.

- A space $X$ is selectively sequentially separable ( $M$-sequentially separable), if $X$ satisfies $S_{f i n}(\mathcal{S}, \mathcal{S})([4$, Definition 1.2]).

For a topological space $X$ we have the next relations of selectors for sequences of dense sets of $X$.

$$
\begin{aligned}
& S_{1}(\mathcal{S}, \mathcal{S}) \Rightarrow S_{f i n}(\mathcal{S}, \mathcal{S}) \Rightarrow S_{f i n}(\mathcal{S}, \mathcal{D}) \Leftarrow S_{1}(\mathcal{S}, \mathcal{D}) \\
& \Uparrow \Uparrow \Uparrow \Uparrow \Uparrow \\
& S_{1}(\mathcal{D}, \mathcal{S}) \Rightarrow S_{\text {fin }}(\mathcal{D}, \mathcal{S}) \Rightarrow S_{\text {fin }}(\mathcal{D}, \mathcal{D}) \Leftarrow S_{1}(\mathcal{D}, \mathcal{D})
\end{aligned}
$$

We write $\Pi\left(\mathcal{A}_{x}, \mathcal{B}_{x}\right)$ without specifying $x$, we mean $(\forall x) \Pi\left(\mathcal{A}_{x}, \mathcal{B}_{x}\right)$.

- A space $X$ has property $\alpha_{2}$ ( $\alpha_{2}$ in the sense of Arhangel'skii), if $X$ satisfies $S_{1}\left(\Gamma_{x}, \Gamma_{x}\right)[1]$.

- A space $X$ has property $\alpha_{4}$ ( $\alpha_{4}$ in the sense of Arhangel'skii), if $X$ satisfies $S_{f i n}\left(\Gamma_{x}, \Gamma_{x}\right)[1]$.

So we have three types of topological properties described through the selection principles:

- local properties of the form $S_{*}\left(\Phi_{x}, \Psi_{x}\right)$;

- global properties of the form $S_{*}(\Phi, \Psi)$;

- semi-local properties of the form $S_{*}\left(\Phi, \Psi_{x}\right)$.

In a series of papers it was demonstrated that $\gamma$-covers, Borel covers, $k$-covers play a key role in function spaces ([5],[10]-[8], [13]-[15], [18]-[25] and many others). We continue to investigate applications of $k$-covers in function spaces with the compact-open topology.

A great attention has recently received the notions of selective separability and selective sequential separability $\left(S_{f i n}(\mathcal{S}, \mathcal{S})\right)[2,3,6,7]$. In this paper, we give characterizations for $C_{k}(X)$ to satisfy $S_{f i n}(\mathcal{S}, \mathcal{S}), S_{f i n}\left(\mathcal{S}, \Gamma_{x}\right)$, and $S_{f i n}\left(\Gamma_{x}, \Gamma_{x}\right)$. 


\section{Main results}

Definition 3.1. A $\gamma_{k}$-cover $\mathcal{U}$ of co-zero sets of $X$ is $\gamma_{k}$-shrinkable if there exists a $\gamma_{k}$-cover $\{F(U): U \in \mathcal{U}\}$ of zero-sets of $X$ with $F(U) \subset U$ for every $U \in \mathcal{U}$.

Note that every $\gamma_{k}$-shrinkable cover contains a countable $\gamma_{k}$-shrinkable cover.

For a topological space $X$ we denote:

- $\Gamma_{k}^{s h}$ - the family of $\gamma_{k}$-shrinkable covers of $X$.

-Similar to the proof that $S_{1}\left(\mathcal{K}, \Gamma_{k}\right)=S_{f i n}\left(\mathcal{K}, \Gamma_{k}\right)$ ([9, Theorem 5]), we prove the following.

Lemma 3.2. For a space $X$ the following are equivalent:

(1) $X$ satisfies $S_{f i n}\left(\Gamma_{k}^{s h}, \Gamma_{k}\right)$;

(2) $X$ satisfies $S_{1}\left(\Gamma_{k}^{s h}, \Gamma_{k}\right)$.

Proof. (1) $\Rightarrow(2)$. Let $\left(\mathcal{U}_{n}: n \in \mathbb{N}\right)$ be a sequence of (countable) $\gamma_{k}$-shrinkable covers of $X$; suppose that for each $n \in \mathbb{N}, \mathcal{U}_{n}=\left\{U_{n, m}: m \in \mathbb{N}\right\}$. Let $V_{n, m}=U_{1, m} \cap \ldots \cap U_{n, m}$ and let $\mathcal{V}_{n}=\left\{V_{n, m}: m \in \mathbb{N}\right\}$. Then $\left(\mathcal{V}_{n}: n \in \mathbb{N}\right)$ is a sequence of $\gamma_{k}$-shrinkable covers of $X$. Since $X$ satisfies $S_{\text {fin }}\left(\Gamma_{k}^{s h}, \Gamma_{k}\right)$ choose for each $n \in \mathbb{N}$ a finite subset $\mathcal{W}_{n}$ of $\mathcal{V}_{n}$ such that $\bigcup_{n \in \mathbb{N}} \mathcal{W}_{n}$ is a $\gamma_{k}$-cover of $X$. (Note that some $\mathcal{W}_{n}$ 's can be empty.)

As $\bigcup_{n \in \mathbb{N}} \mathcal{W}_{n}$ is infinite and all $\mathcal{W}_{n}$ 's are finite, there exists a sequence $m_{1}<m_{2}<\ldots<$ $m_{p}<\ldots$ in $\mathbb{N}$ such that for each $i \in \mathbb{N}$ we have $\mathcal{W}_{m_{i}} \backslash \bigcup_{j<i} \mathcal{W}_{m_{j}} \neq \emptyset$. Choose an element $W_{m_{i}} \in \mathcal{W}_{m_{i}} \backslash \bigcup_{j<i} \mathcal{W}_{m_{j}}, i \in \mathbb{N}$, and fix its representation $W_{m_{i}}=U_{1, k_{m_{i}}} \cap U_{2, k_{m_{i}}} \cap \ldots \cap U_{m_{i}, k_{m_{i}}}$ as above.

Since each infinite subset of a $\gamma_{k}$-cover is also a $\gamma_{k}$-cover, we have that the set $\left\{W_{m_{i}}\right.$ : $i \in \mathbb{N}\}$ is a $\gamma_{k}$-cover of $X$. For each $n \leq m_{1}$ let $U_{n} \in \mathcal{U}_{n}$ be the $n$-th coordinate of $W_{m_{1}}$ in the chosen representation of $W_{m_{1}}$, and for each $n \in\left(m_{i}, m_{i+1}\right], i \geq 1$, let $U_{n} \in \mathcal{U}_{n}$ be the $n$-th coordinate of $W_{m_{i+1}}$ in the above representation of $W_{m_{i+1}}$. Observe that each $U_{n} \supset W_{m_{i+1}}$. Therefore, we obtain a sequence $\left(U_{n}: n \in \mathbb{N}\right)$ of elements, one from each $\mathcal{U}_{n}$, which form a $\gamma_{k}$-cover of $X$ and show that $X$ satisfies $S_{1}\left(\Gamma_{k}^{s h}, \Gamma_{k}\right)$.

The symbol 0 denotes the constantly zero function in $C_{k}(X)$. Because $C_{k}(X)$ is homogeneous we can work with $\mathbf{0}$ to study local and semi-local properties of $C_{k}(X)$.

Theorem 3.3. For a Tychonoff space $X$ the following statements are equivalent:

(1) $C_{k}(X)$ satisfies $S_{1}\left(\Gamma_{\mathbf{0}}, \Gamma_{\mathbf{0}}\right)$ [property $\alpha_{2}$ ];

(2) $X$ satisfies $S_{1}\left(\Gamma_{k}^{s h}, \Gamma_{k}\right)$.

Proof. (1) $\Rightarrow(2)$. Let $\left(\mathcal{U}_{n}: n \in \mathbb{N}\right)$ be a sequence of (countable) $\gamma_{k}$-shrinkable covers of $X$; suppose that for each $n \in \mathbb{N}, \mathcal{U}_{n}=\left\{U_{n, m}: m \in \mathbb{N}\right\}$ and $\left\{F\left(U_{n, m}\right): U_{n, m} \in \mathcal{U}_{n}\right\}$ is a $\gamma_{k}$-cover of zero-sets of $X$ with $F\left(U_{n, m}\right) \subset U_{n, m}$ for every $U_{n, m} \in \mathcal{U}_{n}$. For each $n, m \in \mathbb{N}$ we fix $f_{n, m} \in C(X)$ such that $f_{n, m} \uparrow F\left(U_{n, m}\right) \equiv 0, f_{n, m} \uparrow\left(X \backslash U_{n, m}\right) \equiv 1$. Consider $S_{n}=\left\{f_{n, m}: m \in \mathbb{N}\right\}$. Since $\left\{F\left(U_{n, m}\right): U_{n, m} \in \mathcal{U}_{n}\right\}$ is a $\gamma_{k}$-cover of $X$, then $S_{n} \in \Gamma_{\mathbf{0}}$ for each $n \in \mathbb{N}$. By (1), there is $\left\{f_{n, m(n)}: n \in \mathbb{N}\right\}$ such that $f_{n, m(n)} \in S_{n}$ and $\left\{f_{n, m(n)}: n \in \mathbb{N}\right\} \in \Gamma_{\mathbf{0}}$. We show that $\left\{U_{n, m(n)}: n \in \mathbb{N}\right\} \in \Gamma_{k}$. Suppose $A \in \mathbb{K}(X)$ and $W=\left[A,\left(-\frac{1}{2}, \frac{1}{2}\right)\right]$ is a base neighborhood of $\mathbf{0}$ then there exists $n^{\prime} \in \mathbb{N}$ such that $f_{n, m(n)} \in W$ for every $n>n^{\prime}$. It follows that $A \subset U_{n, m(n)}$ for every $n>n^{\prime}$.

$(2) \Rightarrow(1)$. Let $S_{n} \in \Gamma_{\mathbf{0}}$ for every $n \in \mathbb{N}$; suppose that for each $n \in \mathbb{N}, S_{n}=\left\{f_{n, j}: j \in\right.$ $\mathbb{N}\}$. Consider $\mathcal{V}_{n}=\left\{f_{n, j}^{-1}\left(\left(-\frac{1}{n}, \frac{1}{n}\right)\right): f_{n, j} \in S_{n}\right\}$ for each $n \in \mathbb{N}$.

Let $J=\left\{n \in \mathbb{N}: f_{n, j}^{-1}\left(\left(-\frac{1}{n}, \frac{1}{n}\right)\right)=X\right.$ for some $\left.j \in \mathbb{N}\right\}$. If $J$ is finite, then we can ignore such finitely many $n$. If $J$ is infinite, then for some $j_{n}(n \in J), f_{n, j_{n}} \rightarrow \mathbf{0}$ uniformly. Thus, without loss of generality, we may assume $f_{n, j}^{-1}\left(\left(-\frac{1}{n}, \frac{1}{n}\right)\right) \neq X$ for each $n, j \in \mathbb{N}$.

Note that $\mathcal{W}_{n}=\left\{f_{n, j}^{-1}\left(\left[-\frac{1}{n+1}, \frac{1}{n+1}\right]\right): f_{n, j} \in S_{n}\right\}$ is a $\gamma_{k}$-cover of zero-sets of $X$. Hence, $\mathcal{V}_{n} \in \Gamma_{k}^{s h}$ for each $n \in \mathbb{N}$. By $(2)$, there is $\left\{f_{n, j(n)}: n \in \mathbb{N}\right\}$ such that $\left\{f_{n, j(n)}^{-1}\left(\left(-\frac{1}{n}, \frac{1}{n}\right)\right)\right.$ : 
$n \in \mathbb{N}\} \in \Gamma_{k}$. We show that $\left\{f_{n, j(n)}: n \in \mathbb{N}\right\} \in \Gamma_{\mathbf{0}}$. Let $[A,(-\epsilon, \epsilon)]$ be a base neighborhood of $\mathbf{0}$ where $A \in \mathbb{K}(X)$ and $\epsilon>0$. There is $n^{\prime} \in \mathbb{N}$ such that $A \subset f_{n, j(n)}^{-1}\left(\left(-\frac{1}{n}, \frac{1}{n}\right)\right)$ for each $n>n^{\prime}$. There is $n^{\prime \prime}>n^{\prime}$ such that $\frac{1}{n^{\prime \prime}}<\epsilon$, hence, $f_{n, j(n)} \in\left[A,\left(-\frac{1}{n^{\prime \prime}}, \frac{1}{n^{\prime \prime}}\right)\right] \subset[A,(-\epsilon, \epsilon)]$ for each $n>n^{\prime \prime}$.

Proposition 3.4 ([3, Proposition 4.2]). Every selectively sequentially separable space is sequentially separable.

We shall prove the following theorem under the condition that the space $C_{k}(X)$ is sequentially separable.

Theorem 3.5. For a Tychonoff space $X$ such that $C_{k}(X)$ is sequentially separable the following statements are equivalent:

(1) $C_{k}(X)$ satisfies $S_{1}(\mathcal{S}, \mathcal{S})$;

(2) $C_{k}(X)$ satisfies $S_{1}\left(\mathcal{S}, \Gamma_{\mathbf{0}}\right)$;

(3) $C_{k}(X)$ satisfies $S_{1}\left(\Gamma_{\mathbf{0}}, \Gamma_{\mathbf{0}}\right)$ [property $\left.\alpha_{2}\right]$;

(4) $X$ satisfies $S_{1}\left(\Gamma_{k}^{s h}, \Gamma_{k}\right)$;

(5) $C_{k}(X)$ satisfies $S_{\text {fin }}(\mathcal{S}, \mathcal{S})$ [selectively sequentially separable];

(6) $C_{k}(X)$ satisfies $S_{\text {fin }}\left(\mathcal{S}, \Gamma_{\mathbf{0}}\right)$;

(7) $C_{k}(X)$ satisfies $S_{f i n}\left(\Gamma_{\mathbf{0}}, \Gamma_{\mathbf{0}}\right)$ [property $\alpha_{4}$ ];

(8) $X$ satisfies $S_{f i n}\left(\Gamma_{k}^{s h}, \Gamma_{k}\right)$.

Proof. (1) $\Rightarrow$ (4). Let $\left\{\mathcal{U}_{i}\right\} \subset \Gamma_{k}^{s h}, \mathcal{U}_{i}=\left\{U_{i}^{m}: m \in \mathbb{N}\right\}$ for each $i \in \mathbb{N}$ and let $S=\left\{h_{m}: m \in \mathbb{N}\right\}$ be a countable sequentially dense subset of $C_{k}(X)$.

For each $i, m \in \mathbb{N}$ we fix $f_{i}^{m} \in C(X)$ such that $f_{i}^{m}\left\lceil F\left(U_{i}^{m}\right)=h_{m}\right.$ and $f_{i}^{m} \uparrow\left(X \backslash U_{i}^{m}\right)=$ 1. Let $S_{i}=\left\{f_{i}^{m}: m \in \mathbb{N}\right\}$. Since $S$ is a countable sequentially dense subset of $C_{k}(X)$, we have that $S_{i}$ is a countable sequentially dense subset of $C_{k}(X)$ for each $i \in \mathbb{N}$. Let $h \in C(X)$, there is a set $\left\{h_{m_{s}}: s \in \mathbb{N}\right\} \subset S$ such that $\left\{h_{m_{s}}\right\}_{s \in \mathbb{N}}$ converges to $h$. Let $K$ be a compact subset of $X, \epsilon>0$ and let $W=\langle h, K, \epsilon\rangle$ be a base neighborhood of $h$, then there is a number $m_{0}$ such that $K \subset F\left(U_{i}^{m}\right)$ for $m>m_{0}$ and $h_{m_{s}} \in W$ for $m_{s}>m_{0}$. Since $f_{i}^{m_{s}} \uparrow K=h_{m_{s}} \uparrow K$ for each $m_{s}>m_{0}, f_{i}^{m_{s}} \in W$ for each $m_{s}>m_{0}$. It follows that a sequence $\left\{f_{i}^{m_{s}}\right\}_{s \in \mathbb{N}}$ converges to $h$.

Since $C_{k}(X)$ satisfies $S_{1}(\mathcal{S}, \mathcal{S})$, there is a sequence $\left\{f_{i}^{m(i)}\right\}_{i \in \mathbb{N}}$ such that for each $i$, $f_{i}^{m(i)} \in S_{i}$, and $\left\{f_{i}^{m(i)}: i \in \mathbb{N}\right\}$ is an element of $\mathcal{S}$.

We show that $\left\{U_{i}^{m(i)}: i \in \mathbb{N}\right\}$ is a $\gamma_{k}$-cover of $X$.

There is a sequence $\left\{f_{i_{j}}^{m\left(i_{j}\right)}\right\}$ converges to $\mathbf{0}$. Let $K$ be a compact subset of $X$ and let $U=\langle\mathbf{0}, K,(-1,1)\rangle$ be a base neighborhood of $\mathbf{0}$. Then there exists $j_{0} \in \mathbb{N}$ such that $f_{i_{j}}^{m\left(i_{j}\right)} \in U$ for each $j>j_{0}$. It follows that $K \subset U_{i_{j}}^{m\left(i_{j}\right)}$ for $j>j_{0}$. By Lemma 3.2, $S_{f i n}\left(\Gamma_{k}^{s h}, \Gamma_{k}\right)=S_{1}\left(\Gamma_{k}^{s h}, \Gamma_{k}\right)$.

$(4) \Leftrightarrow(3)$. By Theorem 3.3.

$(3) \Rightarrow(2)$ is immediate.

$(2) \Rightarrow(1)$. For each $n \in \mathbb{N}$, let $S_{n}$ be a sequentially dense subset of $C_{k}(X)$ and let $\left\{h_{n}: n \in \mathbb{N}\right\}$ be sequentially dense in $C_{k}(X)$. Take a sequence $\left\{f_{n}^{m}: m \in \mathbb{N}\right\} \subset S_{n}$ such that $f_{n}^{m} \mapsto h_{n}(m \mapsto \infty)$. Then $f_{n}^{m}-h_{n} \mapsto \mathbf{0}(m \mapsto \infty)$. Hence, there exists $f_{n}^{m_{n}}$ such that $f_{n}^{m_{n}}-h_{n} \mapsto \mathbf{0}(n \mapsto \infty)$. We see that $\left\{f_{n}^{m_{n}}: n \in \mathbb{N}\right\}$ is sequentially dense. Let $h \in C_{k}(X)$ and take a sequence $\left\{h_{n_{j}}: j \in \mathbb{N}\right\} \subset\left\{h_{n}: n \in \mathbb{N}\right\}$ converging to $h$. Then, $f_{n_{j}}^{m_{n_{j}}}=\left(f_{n_{j}}^{m_{n_{j}}}-h_{n_{j}}\right)+h_{n_{j}} \mapsto h(j \mapsto \infty)$.

$(4) \Leftrightarrow(8)$. By Lemma 3.2.

The proofs of the remaining implications are similar to those proved above. 
Recall that the $i$-weight $i w(X)$ of a space $X$ is the smallest infinite cardinal number $\tau$ such that $X$ can be mapped by a one-to-one continuous mapping onto a Tychonoff space of the weight not greater than $\tau$.

It is well known that if $X$ is hemicompact then $C_{k}(X)$ is metrizable. It follows that $C_{k}(X)$ is sequential separable for a hemicompact space $X$ with $i w(X)=\aleph_{0}$. But, for general case, the author does not know the answer to the next question.

Question 1. Characterize a Tychonoff space $X$ such that a space $C_{k}(X)$ is sequential separable?

Proposition 3.6 ([3, Corollary 4.8 (Dow-Barman)]). Every Fréchet-Urysohn separable $T_{2}$ space is selectively separable (hence, selectively sequentially separable).

It is well known that a Tychonoff space $X$ the space $C_{k}(X)$ is Fréchet-Urysohn if and only if $X$ satisfies $S_{1}\left(\mathcal{K}, \Gamma_{k}\right)$ ([11]).

A Tychonoff space $X$ the space $C_{k}(X)$ is separable if and only if $i w(X)=\aleph_{0}[16]$.

Question 2. Is there a Tychonoff space $X$ with $i w(X)=\aleph_{0}$ such that $C_{k}(X)$ satisfies $S_{1}(\mathcal{S}, \mathcal{S})$, but $C_{k}(X)$ is not Fréchet-Urysohn (i.e. $X$ satisfies $S_{1}\left(\Gamma_{k}^{s h}, \Gamma_{k}\right)$, but it has not property $\left.S_{1}\left(\mathcal{K}, \Gamma_{k}\right)\right)$ ?

Acknowledgment. I would like to thank the anonymous referee who read carefully the manuscript and helped me to simplify and improve the presentation of the results of the paper.

\section{References}

[1] A.V. Arhangel'skii, The frequency spectrum of a topological space and the classification of spaces, Soviet Math. Dokl. 13, 1186-1189, 1972.

[2] A. Bella, M. Bonanzinga, and M. Matveev, Variations of selective separability, Topol. App. 156, 1241-1252, 2009.

[3] A. Bella, M. Bonanzinga, and M. Matveev, Sequential+separable vs sequentially separable and another variation on selective separability, Cent. Eur. J. Math. 11 (3), 530-538, 2013.

[4] A. Bella and C. Costantini, Sequential Separability vs Selective Sequential Separability, Filomat 29 (1), 121-124, 2015.

[5] A. Caserta, G. Di Maio, Lj.D.R. Kočinac, and E. Meccariello, Applications of k-covers II, Topol. App. 153, 3277-3293, 2006.

[6] P. Gartside, J.T.H. Lo, and A. Marsh, Sequential density, Topol. App. 130, 75-86, 2003.

[7] G. Gruenhage and M. Sakai, Selective separability and its variations, Topol. App. 158 (12), 1352-1359, 2011.

[8] Lj.D.R. Kočinac, Closure properties of function spaces, App. Gen. Top. 4 (2), 255$261,2003$.

[9] Lj.D.R. Kočinac, $\gamma$-sets, $\gamma_{k}$-sets and hyperspaces, Mathematica Balkanica 19, 109$118,2005$.

[10] Lj.D.R. Kočinac, Selection principles and continuous images, Cubo Math. J. 8 (2), 23-31, 2006.

[11] S. Lin, C. Liu, and H. Teng, Fan tightness and strong Fréchet property of $C_{k}(X)$, Adv. Math. (Chinese) 23 (3), 234-237, 1994.

[12] G.Di Maio, Lj.D.R. Kočinac, and E. Meccariello, Applications of k-covers, Acta Math. Sin. (English Series) 22 (4), 1151-1160, 2006.

[13] G.Di Maio, Lj.D.R. Kočinac, and T. Nogura Convergence properties of hyperspaces, J. Korean Math. Soc. 44 (4), 845-854, 2007. 
[14] A.J. Marsh, Topology of function spaces, Doctoral Dissertation, University of Pittsburgh, 2004.

[15] R.A. McCoy, Function spaces which are k-spaces, Topol. P. 5, 139-146, 1980.

[16] N. Noble, The density character of functions spaces, Proc. Amer. Math. Soc. 42, 228-233, 1974.

[17] A.V. Osipov, Different kinds of tightness of a funtional space, Tr. Inst. Mat. Mekh. (Russian), 22 (3), 192-199, 2016.

[18] A.V. Osipov, Application of selection principles in the study of the properties of function spaces, Acta Math. Hungar. 154 (2), 362-377, 2018.

[19] A.V. Osipov, Classification of selectors for sequences of dense sets of $C_{p}(X)$, Topology Appl. 242, 20-32, 2018.

[20] A.V. Osipov, The functional characterizations of the Rothberger and Menger properties, Topology Appl. 243, 146-152, 2018.

[21] A.V. Osipov, Classification of selectors for sequences of dense sets of Baire functions, submitted.

[22] A.V. Osipov and S. Özçă̆, Variations of selective separability and tightness in function spaces with set-open topologies, Topology Appl. 217, 38-50, 2017.

[23] A.V. Osipov and E.G. Pytkeev, On sequential separability of functional spaces, Topology Appl. 221, 270-274, 2017.

[24] B.A. Pansera and V. Pavlović, Open covers and function spaces, Matematički Vesnik $\mathbf{5 8}, 57-70,2006$.

[25] M. Sakai, k-Frechet-Urysohn Property of $C_{k}(X)$, Topol. App. 154 (7), 1516-1520, 2007.

[26] G. Tironi and R. Isler, On some problems of local approximability in compact spaces, In: General Topology and its Relations to Modern Analysis and Algebra III, 443-446, Prague, August 30-September 3, 1971, Academia, Prague, 1972.

[27] A. Wilansky, How separable is a space?, Amer. Math. Monthly 79 (7), 764-765, 1972. 